Title:

Neutrino Properties And Fundamental Symmetries

Author(s):

T. J. Bowles, P-23

Submitted to:

DOE Office of Scientific and Technical Information (OSTI)

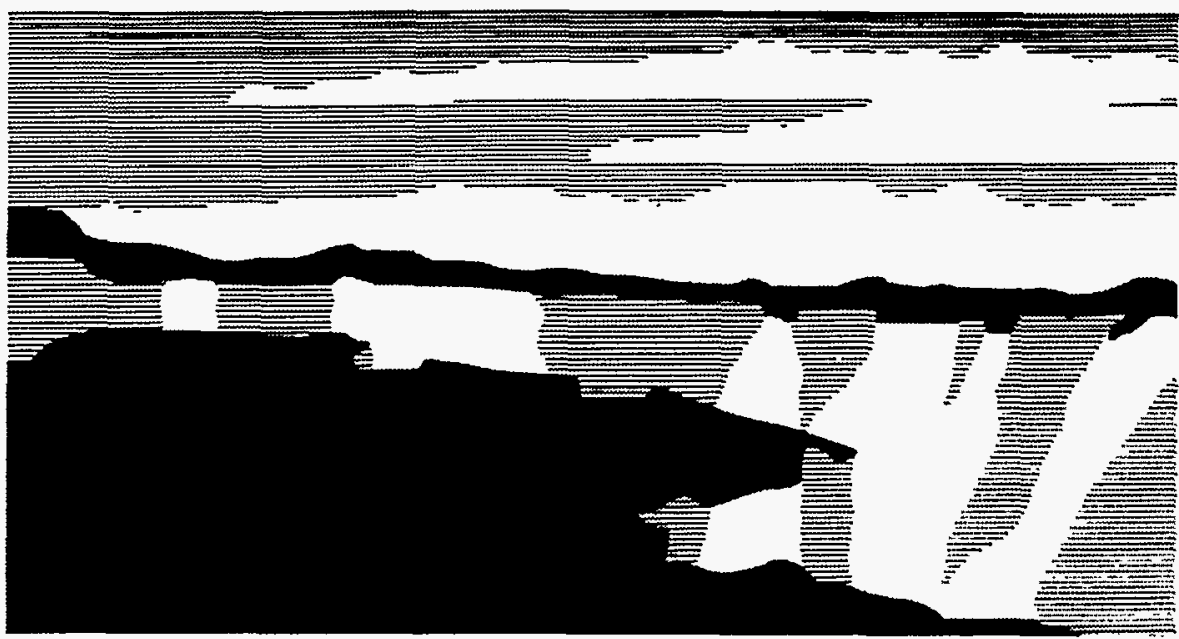

Los Alamos National Laboratory, an affirmative action/equal opportunity employer, is operated by the University of Califomia for the U.S. Department of Energy under contract W-7405-ENG-36. By acceptance of this article, the publisher recognizes that the U.S. Government retains a nonexclusive, royaltyfree license to publish or reproduce the published form of this contribution, or to allow others to do so, for U.S. Government purposes. The Los Alamos National Laboratory requests that the publisher identify this article as work performed under the auspices of they.S Rpatpantangy. 


\title{
Neutrino Properties And Fundamental Symmetries
}

T.J. Bowles*, S. Brice, E.I. Esch, M.M. Fowler, A. Goldschmidt, G. L. Greene, A. Hime, F. McGirt, P. Thornewell, J.B. Wilhelmy, and J.M. Wouters Los Alamos National Laboratory

\author{
K. Lising, S. J. Freedman, and E. Wasserman \\ University of California at Berkeley
}

M. Browne, P. J. Doe, C. Duba, S. R. Elliott, J. Germani, R.G.H. Robertson, A. Rushdy, T. Steiger, and J.F. Wilkerson University of Washington

T. Chupp, K. Coulter, and S. Hwang University of Michigan

S. Dewey, J. Nico, A. Thompson, and F. Wietfeldt National Institute Of Standards And Technology

Y.D. Chan, B. Fujikawa, K.T. Lesko, M. Moorhead, E. Norman, A.R. Smith, and R. Stokstad

Lawrence Berkeley National Laboratory

\author{
A. Garcia \\ Notre Dame University \\ M. Snow \\ Indiana University \\ R. Ollerhead \\ University of Guelph
}

*Principal investigator, e-mail: tjb@lanl.gov 


\begin{abstract}
This is the final report of a three-year, Laboratory-Directed Research and Development (LDRD) project at the Los Alamos National Laboratory (LANL). There are two components to this work. The first is a development of a new detection scheme for neutrinos. The observed deficit of neutrinos from the Sun may be due to either a lack of understanding of physical processes in the Sun or may be due to neutrinos oscillating from one type to another during their transit from the Sun to the Earth. The Sudbury Neutrino Observatory (SNO) is designed to use a water Cerenkov detector employing one thousand tonnes of heavy water to resolve this question. The ability to distinguish muon and tau neutrinos from electron neutrinos is crucial in order to carry out a modelindependent test of neutrino oscillations. We describe a developmental exploration of a novel technique to do this using ${ }^{3} \mathrm{He}$ proportional counters. Such a method offers considerable advantages over the initially proposed method of using Cerenkov light from capture on $\mathrm{NaCl}$ in the SNO. The second component of this work is an exploration of optimal detector geometry for a time-reversal invariance experiment. The question of why time moves only in the forward direction is one of the most puzzling problems in modern physics. We know from particle physics measurements of the decay of kaons that there is a charge-parity symmetry that is violated in nature, implying time-reversal invariance violation. Yet, we do not understand the origin of the violation of this symmetry. To promote such an understanding, we are developing concepts and prototype apparatus for a new, highly sensitive technique to search for time-reversal-invariance violation in the beta decay of the free neutron. The optimized detector geometry is seven times more sensitive than that in previous experiments.
\end{abstract}

\title{
1. Background and Research Objectives
}

Los Alamos has had an ongoing presence in the field of weak interaction physics since 1979 and contributes to the long-term effort to address several of the most pressing questions facing modern physics. A central theme of the work has been the search for effects due to a non-zero neutrino mass and tests of fundamental symmetries of nature.

\section{a. ${ }^{3}$ He Neutrino Detector}


Our interest in recent years has turned to solar neutrinos as a means of searching for a neutrino mass with a sensitivity of up to ten orders of magnitude increase over that obtainable in laboratory experiments. This effort was initially centered around a collaboration (SAGE) to carry out a gallium solar neutrino experiment. This experiment is sensitive to the low-energy flux of neutrinos produced in the dominant energy-generating mechanism of the Sun, namely the fusion of two protons. SAGE produced the first gallium results to verify the deficit seen in earlier experiments, which studied higher energy neutrinos from the Sun. To carry out modelindependent tests of neutrino oscillations as being the source of the solar neutrino problem, we joined the Sudbury Neutrino Observatory (SNO) collaboration. Our primary interest in SNO has been in developing a means to differentiate muon and tau neutrinos from electron neutrinos. As muon and tau neutrinos cannot be produced in the Sun (the temperature of the Sun is too low), an observation of these neutrinos will provide a model-independent solution to the solar neutrino problem.

The SNO detector consists of 1000 tonnes of $99.92 \%$ enriched heavy water surrounded by a shield of 7000 tonnes of purified light water. Solar neutrinos can interact with deuterium nuclei in either of two ways,

$$
\begin{aligned}
& { }^{2} \mathrm{H}+\mathrm{v}_{\mathrm{e}} \rightarrow \mathrm{p}+\mathrm{p}+\mathrm{e}^{-}-1.44 \mathrm{MeV} \quad \text { (Charged current: } \mathrm{CC} \text { ) } \\
& { }^{2} \mathrm{H}+\mathrm{v}_{\mathrm{x}} \rightarrow \mathrm{p}+\mathrm{n}+\mathrm{v}_{\mathrm{x}}-2.22 \mathrm{MeV} \quad \text { (Neutral current: NC) }
\end{aligned}
$$

Because the neutral current process is induced by any active flavor of neutrino, whereas fusion reactions in the sun produce only $v_{\mathrm{e}}$, comparison of these two rates provides direct information about neutrino oscillations, independent of any solar model. SNO will also provide a direct spectrum of electron neutrinos above $6.5 \mathrm{MeV}$, to reveal possible Mikheyev-SmirnovWolfenstein (MSW) resonant neutrino oscillations in matter, which result in a distortion of the energy spectrum.

SNO is being built at a depth of 2200 meters in the International Nickel Company (INCO) Creighton nickel mine in Sudbury, Ontario, by a collaboration of 12 Canadian, US, and UK institutions, including Los Alamos. Initial data taking is anticipated in early 1996. While Los Alamos is responsible for a number of items in SNO, because the NC reaction is of such fundamental importance, we have proposed to detect it by capturing the liberated neutron in ${ }^{3} \mathrm{He}$ proportional counters placed in the heavy water. Such a method offers event-by-event discrimination of NC and CC events, high statistical accuracy, and full-time NC sensitivity in case a supernova should occur.

\section{b. Time-Reversal Invariance Violation Detector Optimization}

The question of why time moves only in the forward direction is one of the most puzzling problems in modern physics. We know from particle physics measurements of the decay of kaons that there is a charge-parity (CP) symmetry that is violated in nature, implying 
time-reversal invariance violation (TRIV), i.e., time has only one direction. Yet we do not understand the origin of the violation of this symmetry. To probe this question, we have pursued an experiment to search for time-reversal invariance violation in the decay of polarized neutrons.

Unlike parity violation, TRIV has only been observed in the kaon system, and presently several alternative theoretical explanations are possible. To untangle this interesting puzzle requires a variety of experiments that have unique sensitivities to the many non-standard models that predict possible TRIV effects. In the beta decay of the free neutron, a non-zero triple correlation between neutron spin, electron momentum, and proton recoil violates time symmetry and singles out effects arising from left-right symmetric physics, leptoquarks, or exotic fermions. Initial work at Los Alamos on exploring detector optimization for this experiment has resulted in a detector geometry potentially seven times more sensitive than geometries employed in previous experiments. The EMIT experiment as designed is expected to reach a sensitivity of $3 \times 10^{-4}$ at the National Institute of Standards and Technology (NIST) reactor, a level that is ten times more sensitive than previous measurements. At this level of sensitivity, the new detector geometry will provide the most stringent constraints of any experiment on some of the $\mathrm{CP}$-violating parameters.

\section{Importance to LANL's Science and Technology Base and National R\&D Needs}

A model-independent proof of neutrino oscillations by SNO would constitute the first observation of the long-sought new physics beyond the Standard Model and would constitute one of the most important scientific measurements of the decade. The identification of the origin of time-reversal violation would solve one of the outstanding puzzles in modern physics and would attract great international interest.

Our group has a strong background in fundamental physics measurements. We have led the US effort in the SAGE solar neutrino experiment and the EMIT experiment. We are recognized internationally for our work on setting a limit on neutrino mass from tritium beta decay. We have extensive experience in dealing with very low background detectors and low rate experiments. Within the Laboratory there is expertise in nuclear and particle physics, chemistry, Monte Carlo methods, development of solid state and proportional counters and fast electronics, fast digitization techniques, and a wide array of ultra-sensitive analysis technologies.

These capabilities have been applied to a number of programmatic efforts at the Laboratory, including Comprehensive Test Ban Treaty verification using neutrino detection, 
the LIDAR project for Desert Storm, and hardware and software development for nonproliferation and counter-proliferation projects. In addition, we have demonstrated the capability to radioassay materials for the computer industry with a sensitivity that is 100 times greater than commercially available. This is a critical need in the industry as single-bit upset events caused by natural radioactivity are preventing the industry from advancing to the next generation, higher density, very-large-scale-integration fabrication.

\section{Scientific Approach and Results}

\section{a. ${ }^{3}$ He Neutrino Detector}

We have carried out a research and development program to demonstrate the feasibility of using ultra-low background ${ }^{3} \mathrm{He}$ proportional counters to detect the existence of muon and tau neutrinos from the Sun as part of our collaboration in SNO. Counters of this type offer unique advantages over other techniques. The primary difficulties are the requirements that the counters introduce no appreciable level of radioactivity into the SNO detector and that they have a very low internal background and extremely low noise. As the heavy water in the SNO detector must be free of $U$ and $T h$ at the $10^{-14}$ level and the expected detected neutron signal is only about four events per day, these requirements are obviously very stringent. We have developed a number of novel techniques to meet these requirements. This work consists of prototyping counters made of ultra-pure nickel bodies using chemical vapor deposition (CVD), along with a variety of possible means to read out both position and rise time information from the counters.

The ${ }^{3} \mathrm{He}$ counters for neutral current detection in SNO must not only have very low external and internal backgrounds, but they must also have high neutron detection efficiency, cause no substantial interference with the Cerenkov light in the SNO detector, and be able to survive immersion in the heavy water for a period of 10 years. The internal background rates must be reduced by a factor of 100 below the best obtained by any other group. We have investigated a variety of possible solutions to these requirements and have shown that ${ }^{3} \mathrm{He}$ counters made primarily of ultra-pure $\mathrm{Ni}$ will be satisfactory. Other required components (feed throughs, etc.) have been identified with acceptably low radioactivity. We have tested the performance of prototype counters at our low-background laboratory located at the Waste Isolatiuon Pilot Project (WIPP).

We have demonstrated that the design requirements for the detectors can be met. $U$ and Th concentrations in the Ni are within our requirements for most of the samples tested. Corrosion in the heavy water has been shown to be acceptably low. Mechanical designs have been worked out which are considerably simpler than earlier designs. Low noise preamplifiers 
and the associated electronics have been developed. Optimization of the counter gas fill and HV has been carried out. The internal background in the counters appears to be due primarily to the presence of ${ }^{210}$ Po on the surface of the counters. This may be due to either deposition from atmospheric $\mathrm{Rn}$ or to migration of Po from some of the solder used in fabricating the prototype counters. Data on prototype counters taken at our WIPP low-background counting facility has demonstrated levels of intrinsic alpha activity that are 250 times lower than achieved elsewhere. A laser welder/cutter has been purchased which allows us to eliminate the solder used to join the Ni tubes to the endcaps in the earlier prototypes. Initial studies of pulse propagation down the counters has been carried out along with extensive studies of background rejection using pulse waveform information. Work on neutron calibration sources has been carried out. Monte Carlo studies of the optimal distribution of the counters within the heavy water have been completed. A Remotely Operated Vehicle (ROV), which is a small submersible vehicle, has been obtained and tests of deployment of the ${ }^{3} \mathrm{He}$ counters in a small mockup of the SNO detector have been carried out. Thus, essentially all of the questions concerned with construction, deployment, and use of the ${ }^{3} \mathrm{He}$ counters have been successfully addressed.

A Department of Energy (DOE) technical feasibility review panel was convened in March 1995. Their report was very favorable and concluded that we had demonstrated technical feasibility and that DOE should fund construction of the full NC detector array. Subsequently, DOE released $\$ 2.5 \mathrm{M}$ of capital construction funds for NC detector construction.

\section{b. Time-Reversal Invariance Violation Detector Optimization}

In our EMIT experiment, we have found a novel geometry that provides an effective increase by a factor of seven in counting rate. The eight-fold symmetry in the detector design ensures that systematic uncertainties are substantially below that in previous experiments. We have built full-scale prototypes of the proton detectors and an approximate mockup of the beta detectors that will be employed in the EMIT detector. A beam line with some shielding and the mock-up detector was installed at the NIST reactor and a number of test runs were carried out. The detector performance was approximately as expected. These studies verified that the backgrounds need to be reduced and a number of sources of background were identified. Nonetheless, a clear proton signal in coincidence with the beta detector was observed with a signal-to-background ratio of more than three. With this experience in hand, we have optimized the detector performance and expect to be able to substantially reduce backgrounds.

Following completion of our test runs at NIST, we submitted a proposal to DOE in FY94 for $\$ 300 \mathrm{~K}$ in capital funds to build the full scale EMIT detector. This proposal was approved and construction of the detector is almost complete. Los Alamos has had primary responsibility for overall design integration of the detector and beam lines. We will participate 
fully in the setup and running of the experiment at NIST, which will be funded by DOE. We also expect to play a strong role in the analysis of the data and will continue to be involved in optimizing the experiment design as new information becomes available from the runs in FY96. With several months of running at NIST, we expect to be able to reach our projected sensitivity of $3 \times 10^{-4}$. By moving to the $\mathbb{L} \mathrm{L}$ reactor in Grenoble in a second stage experiment, we expect to reach an ultimate sensitivity of $1 \times 10^{-4}$.

\section{Publications}

1. Walston, S., "Calibration of the SNO PMTs through the NCD Array," SNO-STR-94006, 1994.

2. Doe, P.J., Hime, A., Robertson, R.G.H., and Wilhelmy, J.B., "Calibration and Neutral-Current Detectors II," SNO-STR-94-004, 1993.

3. Miller, G.G., Olivares, J.A., and Wilhelmy, J.B., "Determination of Th and U in High Purity Ni," SNO-STR-93-054, 1993.

4. Brewer, B. and Doe, P., "Measurement of Airborne Dust at the Reynolds Polymer Facility in California," SNO-STR-93-053, 1993.

5. Wouters, J., "Status of Rope Acquisition," SNO-STR-93-048, 43, 1993.

6. Poon, A. and Robertson, R.G.H., "Schedule for the LANL ${ }^{3} \mathrm{H}$ (p,gamma) ${ }^{4} \mathrm{He}$ Calibration Source," SNO-STR-93-047, 1993.

7. Robertson, R.G.H., "Neutral-Current Detector Progress Report," SNO-STR-93-046, 1993.

8. Poon, A., "Progress Report on the ${ }^{3} \mathrm{H}\left(\mathrm{p}\right.$,gamma) ${ }^{4} \mathrm{He}$ Calibration Source," SNO-STR93-030, 1993.

9. Walston, S., "Crazing of Stressed Acrylic Induced by Radiacwash and Boric Acid," SNO-STR-93-029, 1993.

10. Wilhelmy, J.B., "Neutral Current Detector Response and Calibration," SNO-STR-93015, 1993.

11. Doe, P., Robertson, R.G.H., and Wilkerson, J.F., "Calibration Source Deployment Inside the Acrylic Vessel," SNO-STR-93-014, 1993.

12. Bowles, T.J., "Solar Neutrino Experiments," 1994 Yearbook of Science and Technology, McGraw-Hill, p 359, 1994.

13. Wilkerson, J.F., "Neutrino," McGraw-Hill Encyclopedia of Science and Technology, McGraw-Hill, 1994. 
14. Bowles, T.J. and Gavrin, V.N., "The Status of the Solar Neutrino Problem," Annu. Rev. Nucl. Part. Sci, 43, 117 (1993).

15. Hime, A., "Do Scattering Effects Resolve the 17-keV Conundrum?," Phys. Lett. B, 299, 165 (1993). 